functional sequelae (Baram TZ. Editorial, Ann Neurol Dec 2003;54:701-704; Millichap JG. Studies in febrile seizures I. Height of body tempeature as a measure of the febrile seizure threshold. Pediatrics Jan 1959;23:76-85).

Effects of seizure-related activity on cognitive function. Paroxysmal epileptic activity, the acute effects of minor seizures and epileptic EEG discharges, caused deficits in cognitive processes, alertness and mental speed, in children with short nonconvulsive seizures, and over time, affected educational achievement (Aldenkamp A, Arends J. Epilepsia January 2004;45:54-63).

\title{
RISK FACTORS FOR RECURRENCE AFTER FIRST SEIZURE
}

Recurrence after a first unprovoked cryptogenic/idiopathic seizure was studied in 213 children followed at FCM-Unicamp, Campinas, SP, Brazil. Recurrence occurred in 34\% of patients at a mean interval of 12 months. An abnormal EEG was a significant risk factor for seizure recurrence. Small calcifications found in $9.5 \%$ of CTs performed in 182 patients were not a predictor for recurrence. (Scotoni AE, Manreza MLG, Guerreiro MM. Recurrence after a first unprovoked cryptogenic/idiopathic seizure in children: a prospective study from Sao Paulo, Brazil. Epilepsia February 2004;45:166-170). (Reprints: Dr MM Guerreiro, Department of Neurology, FCM-Unicamp, PO Box 6111, 13083-970 Campinas, SP, Brazil).

COMMENT. EEG but not $\mathrm{CT}$ abnormalities are predictors of seizure recurrence after a first unprovoked cryptogenic/idiopathic seizure.

In 82 patients with childhood-onset cryptogenic localization-related epilepsies controlled for 3 years or more, seizures recurred in $8(9.8 \%)$ after withdrawal of antiepileptic drugs. (Ohta H et al. Brain Dev 2004;26:19-25). Factors correlating with higher rates of seizure relapse included: 6 years of age or higher at epilepsy onset; 15 years of age or higher at start of AED withdrawal; 5 years or more from start of AED treatment to seizure control; 5 or more seizures before seizure control; and 2 or more AED to effect control. Independent risk factors for relapse were: 6 years of age or higher at onset, and 5 years or more from start of treatment to seizure control. These risk factors are of value in attempting AED withdrawal.

\section{ATTENTION DEFICIT DISORDERS}

\section{EFFECTS OF METHYLPHENIDATE ON ATTENTION IN ADHD}

In a randomized, double-blind, placebo-controlled study of 60 children aged 8-12 years with ADHD treated with two doses of methylphenidate (MPH), 0.25 and $0.5 \mathrm{mg} / \mathrm{kg}$, and placebo, a linear improvement was obtained at both doses in alertness and focused and sustained attention. No significant improvement occurred for divided attention. Intensitydimension functions are best influenced by higher doses, executive functions by moderate doses, and selective-dimension functions by variable doses. Responders defined by improved behavior did not differ from nonresponders. (Konrad K, Gunther T, Hanisch C, HerpertzDahlmann B. J Am Acad Child Adolesc Psychiatry Feb 2004;43:191-298). 
2004;43:191-298). (Respond: Dr Konrad, Department of Child and Adolescent Psychiatry, University Hospital Aachen, Neuenhofer Weg 21, D-52074 Aachen, Germany).

COMMENT. Multiple indices of efficacy should be monitored during treatment of $\mathrm{ADHD}$, and the dosage of MPH selected according to the targeted area, inattention or hyperactivity-impulsivity. The clinical practice of determining response to stimulant therapy from parent or teacher rating may not be sufficient, and measures of several components of attention (sustained, focused, divided, stop-signal paradigm) should be considered when practical and available.

Antihistamine side-effects in children with allergic rhinitis. Antihistamines are frequently taken by children with ADHD and are sometimes considered to aggravate the symptoms. Cetrizine $10 \mathrm{mg}$, a second-generation antihistamine, increased P300 latency and had a sedative effect without a significant change in subjective somnolence, as measured by a visual analog scale. (Ng KH et al. Pediatrics February 2004;113:e116-e121). Repeated dosing may be found to adversely affect attention, and antihistamines should be considered a potential risk factor for impaired learning.

\section{HEADACHE DISORDERS}

\section{ADVANCES IN MIGRAINE MECHANISMS AND TREATMENT}

Migraine mechanisms are discussed in relation to familial hemiplegic migraine (FHM) genotypes by investigators from the Massachusetts General Hospital, Boston, and Universities in Ankara, Turkey. FHM, a dominantly inherited disease, is characterized by sustained attacks of visual, somatosensory, and aphasic auras followed by motor weakness or paralysis. FHM type 1 mutation exhibits cerebellar signs, but otherwise types 1 and 2 FHM cannot be easily distinguished phenotypically. Cortical spreading depression (CSD) causes migraine aura, and glutamate triggers CSD. FHM mutations render the brain more susceptible to prolonged CSD by excessive synaptic glutamate release (type 1) or decreased removal of glutamate and $\mathrm{K}$ from the synaptic cleft (type 2). (Moskowitz MA, Bolay H, Dalkara T. Deciphering migraine mechanisms: Clues from familial hemiplegic migraine phenotypes. Ann Neurol February 2004;55:276-280). (Respond: Michael A Moskowitz MD, Neuroscience Center, Department of Radiology and Neurology, Massachusetts General Hospital, Harvard Medical School, $14913^{\text {th }}$ Street, Room 6403, Charlestown, MA 02129).

COMMENT. Migraine pathogenesis and possible mechanisms of action of preventive therapies are reviewed by Welch KMA. (Neurology Oct 2003;61(Suppl 4):S2-S8). A cerebral cortical origin of migraine aura, cortical hyperexcitability and CSD, and the trigeminovascular system and its central projections are involved in the migraine attack. Progressive damage to the periaqueductal gray matter (PAG) by iron deposition may explain change in phenotypic expression and why episodic migraine becomes chronic over time in some patients. Antimigraine mechanisms include Na channel blockade (amitryptyline), intracellular $\mathrm{Ca}$ modulation (gabapentin), blockade of NMDA receptors (magnesium), aminergic-mediated modulation (propranolol), GABA inhibition potentiation (topiramate), and GABA-mediated inhibition of cell excitation (valproate). 